\title{
ON WAVES DUE TO SMALL OSCILLATIONS OF A VERTICAL PLATE SUBMERGED IN DEEP WATER
}

\author{
B. N. MANDAL ${ }^{1}$
}

(Received 15 May 1989)

\begin{abstract}
This paper is concerned with surface water waves produced by small oscillations of a thin vertical plate submerged in deep water. Green's integral theorem in the fluid region is used in a suitable manner to obtain the amplitude for the radiated waves at infinity. Particular results for roll and sway of the plate, and for a line source in the presence of a fixed vertical plate, are deduced.
\end{abstract}

\section{Introduction}

Ursell [8] used Havelock's expansion of the water wave potential to obtain an explicit expression for the wave amplitude at infinity for the wave motion set up by small oscillations of a partially immersed thin vertical plate due to rolling about a horizontal axis in its plane. Evans [3] used Green's integral theorem in the fluid region in a very interesting manner to obtain the amplitude (complex) of the radiated wave at infinity produced by the general motion of a partially immersed flexible plate and recovered Ursell's [8] result for a rolling ship as a special case. He also deduced expressions for the wave amplitude at infinity due to sway of the plate, given by Haskind [5], and due to a line source situated in the fluid in the presence of a fixed vertical partially immersed plate. Recently Basu and Mandal [1] used Evans' [3] idea to derive the effect of a completely submerged fixed vertical plane barrier on the amplitude at infinity of the waves radiated by a line source. Wave amplitudes at infinity for these problems were derived in a natural

\footnotetext{
${ }^{1}$ Physical and Earth Sciences Division, Indian Statistical Institute, Calcutta-700035, India.

(C) Copyright Australian Mathematical Society 1991, Serial-fee code 0334-2700/91
} 
manner by a suitable application of Green's integral theorem in the fluid region, by using Ursell's [7] explicit solutions for the velocity potential when a two-dimensional plane wave is normally incident upon a fixed vertical plane barrier partially immersed or completely submerged in deep water.

The problem of a two-dimensional plane wave normally incident upon a fixed vertical submerged plate was considered by Evans [2], who solved it by a technique based on the theory of complex variables. However, the explicit form of the velocity potential was not given by Evans [2]. Mandal and Goswami [6] considered the problem of a plane wave obliquely incident on the submerged plate, by an integral equation formulation based on Green's integral theorem. From the results of this paper, the explicit form of the velocity potential when a plane wave is normally incident upon the plate can be deduced. In the present paper, this explicit solution for the velocity potential is utilised in Green's integral theorem, in a manner described in the note of Evans [3], to obtain the wave amplitude at infinity produced by the general motion of a completely submerged flexible vertical plate. When the upper edge of the plate approaches the free surface, the various results given by Evans [3] are recovered.

\section{Statement and formulation}

We consider the plane vertical thin plate submerged in deep water which is incompressible and inviscid, and use a coordinate system in which the $y$-axis is taken vertically downwards, the mean free surface is the plane $y=0$ and the position of the plate in the equilibrium position is $x=0, a \leq y \leq b$. The plate makes small oscillations of circular frequency $\sigma$ about its equilibrium position. Assuming the resulting motion in the fluid to be irrotational, a velocity potential exists which can be described by $\operatorname{Re}\{\varphi(x, y) \exp (-i \sigma t)\}$ as the motion will be everywhere harmonic of frequency $\sigma$. Within the framework of the linearised theory of water waves, $\varphi(x, y)$ satisfies

$$
\begin{aligned}
& \nabla^{2} \varphi=0 \quad \text { in the fluid region, } \\
& K \varphi+\varphi_{y}=0 \quad \text { on } y=0
\end{aligned}
$$

where $K=\sigma^{2} / g, g$ being gravity,

$$
\varphi_{x}=f(y) \quad \text { on } x=0, a<y<b,
$$

where $f(y)$ is the prescribed normal velocity of the plate. Thus $\varphi(x, y)$ is odd in $x$. As $|x| \rightarrow \infty$, the waves travel away from the plate so that

$$
\varphi(x, y) \sim \pm A \exp (-K y \pm i K x) \quad \text { as } x \rightarrow \pm \infty
$$


where $A$ is the complex amplitude of the radiated waves at infinity and is to be determined. Near the two edges of the plate, $\nabla \varphi$ has integrable singularities which can be expressed as

$$
r^{1 / 2} \nabla \varphi \rightarrow 0 \quad \text { as } r \rightarrow 0,
$$

where $r$ denotes the distance from the edges $(0, a)$ and $(0, b)$ of the plate. Finally, we assume that

$$
\varphi, \nabla \varphi \rightarrow 0 \quad \text { as } y \rightarrow \infty .
$$

\section{Solution of the problem}

To determine the complex amplitude $A$ of the radiated waves at infinity, we require the function $\Psi(x, y)$ which is the velocity potential due to a progressive wave train represented by $\exp (-K y+i K x)$ incident upon a fixed vertical submerged plate $x=0, a \leq y \leq b$, from negative infinity. The explicit form of $\Psi(x, y)$ can be deduced from the general result given by Evans [2]. However we deduce it here for completeness from the result given by Mandal and Goswami [6] in the form

$$
\Psi(x, y)=\exp (-K y+i K x)+\Psi_{0}(x, y)
$$

with

$$
\Psi_{0}(x, y)=\frac{1}{2 \pi} \int_{a}^{b} f_{0}(\eta) G_{\xi}(0, \eta ; x, y) d \eta
$$

where

$$
\begin{gathered}
G(\xi, \eta ; x, y)=2 \pi i \exp \{-K(\eta+y)+i K|\xi-x|\} \\
+2 \int_{0}^{\infty} \frac{(k \cos k \eta-K \sin k \eta)(k \cos k y-K \sin k y)}{k\left(k^{2}+K^{2}\right)} e^{-k|\xi-x|} d k \\
f_{0}(\eta)=C \exp \left((-k \eta) \int_{a}^{\eta} \exp (K u) l(u) d u\right. \\
l(u)=\left(d^{2}-u^{2}\right)\left\{\left(u^{2}-a^{2}\right)\left(b^{2}-u^{2}\right)\right\}^{-1 / 2} \\
C=2 i / \Delta, \quad \Delta=\alpha-\beta-i \gamma \\
\alpha=\int_{-a}^{a} \exp (-K u)\left(d^{2}-u^{2}\right)\left\{\left(a^{2}-u^{2}\right)\left(b^{2}-u^{2}\right)\right\}^{-1 / 2} d u \\
\gamma=\int_{a}^{b} \exp (-K u) l(u) d u \\
\beta=\int_{b}^{\infty} \exp (-K u) l(u) d u
\end{gathered}
$$


and $d^{2}$ is given by

$$
\int_{a}^{b} \exp (K u) l(u) d u=0
$$

Using (3.3) in (3.2), $\Psi(x, y)$ can be calculated explicitly for $x>0$ and $x<0$. Equation (3.2) involves two integrals which can be simplified. One integral is $2 K \int_{a}^{b} \exp (-K \eta) f_{0}(\eta) d \eta$ and this is $2 i \gamma / \Delta$, and the other involves $\int_{a}^{b} f_{0}(\eta)(k \cos k \eta-K \sin k \eta) d \eta$ which, after using (3.4), is simplified to $-2 i J(k) / \Delta$ where

$$
J(k)=\int_{a}^{b} l(u) \sin k u d u .
$$

Hence we finally obtain

$$
\begin{aligned}
\Psi(x, y)= & \frac{\alpha-\beta}{\Delta} \exp (-K y+i K x) \\
& -\frac{2 i}{\pi \Delta} \int_{0}^{\infty} \frac{J(k) e^{-k x}(k \cos k y-K \sin k y)}{k^{2}+K^{2}} d k, \text { for } x>0
\end{aligned}
$$

and

$$
\begin{aligned}
\Psi(x, y)= & \exp (-K y+i K x)-\frac{i \gamma}{\Delta} \exp (-K y-i K x) \\
& +\frac{2 i}{\pi \Delta} \int_{0}^{\infty} \frac{J(k) e^{k x}(k \cos k y-K \sin k y)}{k^{2}+K^{2}} d k, \quad \text { for } x<0 .
\end{aligned}
$$

We now apply Green's integral theorem to the harmonic functions $\varphi, \Psi$ within the region bounded by the lines $y=0,-X \leq x \leq X ; x=X$, $0 \leq y \leq Y ; y=Y,-X \leq x \leq X ; x=-X, 0 \leq y \leq Y ; x=0^{+}$, $a \leq y \leq b ; x=0^{-}, a \leq y \leq b$; with $X, Y>0$ and ultimately allow $X, Y$ to tend to infinity. Following the arguments similar to those of Evans [3], we find

$$
A=i \int_{a}^{b} \bar{\Psi}(y) f(y) d y
$$

where

$$
\bar{\Psi}(y)=\Psi(+0, y)-\Psi(-0, y) .
$$

Using (3.12) and (3.13), this gives

$$
\bar{\Psi}(y)=\frac{2 i \gamma}{\Delta} \exp (-K y)-\frac{4 i}{\pi \Delta} \int_{0}^{\infty} \frac{J(k)(k \cos k y-K \sin k y)}{k^{2}+K^{2}} d k .
$$


Now

$$
\begin{aligned}
K \bar{\Psi}(y)+\bar{\Psi}^{\prime}(y) & =\frac{4 i}{\Delta \pi} \int_{0}^{\infty} J(k) \sin k y d k \\
& =\frac{4 i}{\Delta \pi} \int_{a}^{b} l(u)\left\{\int_{0}^{\infty} \sin k u \sin k y d k\right\} d u \\
& =\frac{2 i}{\Delta} \int_{a}^{b} l(u) \delta(u-y) d u \\
& = \begin{cases}\frac{2 l}{\Delta} l(y) & a<y<b \\
0 & \text { otherwise. }\end{cases}
\end{aligned}
$$

Hence

$$
\bar{\Psi}(y)=\frac{2 i}{\Delta} \exp (-K y) \int_{a}^{y} l(u) \exp (K u) d u
$$

where we have used the condition that $\bar{\Psi}(a)=0$, while $\bar{\Psi}(b)=0$, is automatically satisfied due to (3.10). Using (3.17) in (3.14) we find

$$
A=\frac{2}{\Delta} \int_{0}^{b} l(y) g(y) d y
$$

where

$$
g(y)=\exp (K y) \int_{a}^{y} f(t) \exp (-K t) d t .
$$

Equation (3.18) is the general expression for the complex amplitude of waves radiated to infinity due to a prescribed normal velocity $f(y)$ of the plate.

\section{Special cases}

\section{(a) Rolling of a plate}

Let us suppose that the plate is hinged at the point $(0, c)$ and makes small rolling oscillations of amplitude $\theta$ and frequency $\sigma$. Then the plate communicates its normal velocity to the adjacent water particles so that the linearised condition to be satisfied on the plate is

$$
\operatorname{Re}\left\{\varphi_{x} \exp (-i \sigma t)\right\}=i \sigma \theta(c-y) \sin \sigma t \quad \text { on } x=0, a<y<b
$$

which gives

$$
f(y)=-i \sigma \theta(c-y)
$$

Hence by (3.19) we find

$$
g(y)=-\left(i \sigma \theta / K^{2}\right)[1-K(c-y)-\{1-K(c-a)\} \exp \{K(y-a)\}] .
$$

Then

$$
A=-\frac{2 i \sigma \theta}{\Delta K^{2}}\left[\frac{1-K c}{b}\left\{d^{2} K(q)-b^{2} E(q)\right\}+K \frac{\pi}{2}\left(d^{2}-\frac{a^{2}+b^{2}}{2}\right)\right]
$$


after using (3.10), where $K(q)$ and $E(q)$ are complete elliptic integrals (Gradsteyn and Ryzhik [4]) defined by

$$
\left.\begin{array}{rl}
K(q) & =\int_{0}^{\pi / 2}\left(1-q^{2} \sin ^{2} \theta\right)^{-1 / 2} d \theta \\
E(q) & =\int_{0}^{\pi / 2}\left(1-q^{2} \sin ^{2} \theta\right)^{1 / 2} d \theta \\
q & =\left(b^{2}-a^{2}\right)^{1 / 2} / b<1 .
\end{array}\right\}
$$

The corresponding result for an immersed plate given by Evans [3] or Ursell [8] can be deduced from (4.2) if we make $\mu(=a / b) \rightarrow 0$. We note that as $\mu \rightarrow 0$, i.e. $q \rightarrow 1$ (Gradstein and Ryzhik [4], Evans [2]) we have

$$
\begin{aligned}
K(q) & \sim \ln (4 / \mu), E(q) \sim 1, \alpha \sim 0, \\
\beta & \sim-b K_{1}(K b), \gamma \sim b \pi I_{1}(K b) \\
d^{2} & \sim b^{2}\left[1+\frac{\pi}{2}\left\{I_{1}(K b)+L_{1}(K b)\right\}\right] / \ln (4 / \mu)
\end{aligned}
$$

so that as $\mu \rightarrow 0$,

$$
\left.\begin{array}{rl}
\Delta \sim b K_{1}(K b)-i b \pi & I_{1}(K b), \\
d^{2} K(q)-b^{2} E(q) & \sim\left(d^{2} \ln (4 / \mu)-b^{2}\right) \\
& \sim \frac{\pi}{2}\left\{I_{1}(K b)+L_{1}(K b)\right\}
\end{array}\right\}
$$

where $I_{1}(z)$ and $K_{1}(z)$ are modified Bessel functions and $L_{1}(z)$ is a modified Struve function. Hence as $\mu \rightarrow 0$,

$$
A \sim \frac{\sigma \theta b \pi}{\pi I_{1}(K b)+i K_{1}(K b)}\left[\frac{1}{2}-\frac{1-K c}{K b}\left\{I_{1}(K b)+L_{1}(K b)\right\}\right] .
$$

This expression agrees with that obtained by Evans [3] and Ursell [8].

\section{(b) Swaying of a plate}

The amplitude of the waves at infinity due to a swaying plate can be obtained from (4.2) by letting $\theta \rightarrow 0, c \rightarrow \infty$ in such a way that the product $\theta c$ remains a finite real constant $\Theta$, the amplitude of the sway.

$$
A=\frac{2 i \sigma \Theta}{\Delta K b}\left\{d^{2} K(q)-b^{2} E(q)\right\} \text {. }
$$

Haskind's [5] result (also mentioned by Evans [3]) for the case of a partially immersed plate can be deduced as before by making $\mu \rightarrow 0$ in (4.7).

(c) A line source in the presence of a fixed vertical submerged plate

Let a line source be situated at the point $(\xi, \eta)$ in the presence of a fixed vertical submerged plate occupying the position $x=0, a \leq y \leq b$. To obtain the amplitude of the waves at infinity we write

$$
\Psi(x, y)=\varphi+G(x, y, \xi ; \eta)
$$


where $G$ is the potential due to a line source at the point $(\xi, \eta)$ in the absence of the plate and is given by (3.3). Since $\Psi_{x}=0$ on the plate we have

$$
\varphi_{x}(0, y)=-G_{x}(0, y ; \xi, \eta) \quad(a<y<b)
$$

so that

$$
\begin{aligned}
f(y)= & -2 \operatorname{sgn} \xi[\pi K \exp \{-K(y+\eta)+i K|\xi|\} \\
& \left.+\int_{0}^{\infty} \frac{(k \cos k y-K \sin k y)(k \cos k \eta-K \sin k \eta)}{k^{2}+K^{2}} \exp (-k|\xi|) d k\right]
\end{aligned}
$$

Hence

$$
\begin{aligned}
& g(y)=-2 \operatorname{sgn} \xi\left[\frac{\pi}{2} \exp (\right.-K \eta+ \\
&+\int_{0}^{\infty} \frac{k \cos k \eta-K \mid))\{\exp (K(y-2 a))-\exp (-K y)\}}{k^{2}+K^{2}} \exp (-k|\xi|) \\
&\quad \times\{\sin k y-\exp (K(y-a)) \sin k a\} d k] .
\end{aligned}
$$

Using (4.9) in (3.18) we obtain for this case after simplification

$$
\begin{aligned}
A=A(\xi, \eta)= & -\frac{2}{\Delta} \operatorname{sgn} \xi\left[2 \int_{0}^{\infty} \frac{J(k)(k \cos k \eta-K \sin k \eta)}{k^{2}+K^{2}} \exp (-k|\xi|) d k\right. \\
& -\pi \gamma \exp (-K \eta+i K|\xi|)] .
\end{aligned}
$$

Thus if

$$
\Psi(x, y) \sim B^{ \pm}(\xi, \eta) \exp (-K y \pm i K x) \quad \text { as } x \rightarrow \infty
$$

then

$$
\left.\begin{array}{l}
B^{+}(\xi, \eta)=2 \pi i \exp (-K \eta-i K \xi)+A(\xi, \eta), \\
B^{-}(\xi, \eta)=2 \pi i \exp (-K \eta+i K \xi)-A(\xi, \eta) .
\end{array}\right\}
$$

From (3.12) and (3.13) we note that

$$
\begin{aligned}
\Psi(+0, y)= & \frac{\alpha-\beta}{\Delta} \exp (-K y) \\
& -\frac{2 i}{\pi \Delta} \int_{0}^{\infty} \frac{J(k)(k \cos k y-K \sin k y)}{k^{2}+K^{2}} d k
\end{aligned}
$$

and

$$
\begin{aligned}
\bar{\Psi}(-0, y)= & \frac{\alpha-\beta-2 i \gamma}{\Delta} \exp (-K y) \\
& +\frac{2 i}{\pi \Delta} \int_{0}^{\infty} \frac{J(k)(k \cos k y-K \sin k y)}{k^{2}+K^{2}} d k
\end{aligned}
$$


Hence

$$
\begin{aligned}
\bar{\Psi}(y)= & \frac{2 i \gamma}{\Delta} \exp (-K y) \\
& -\frac{4 i}{\pi \Delta} \int_{0}^{\infty} \frac{J(k)(k \cos k y-K \sin k y)}{k^{2}+K^{2}} d k .
\end{aligned}
$$

But $\bar{\Psi}=0$ for $y \leq a$ or $y \geq b$ so that we obtain

$$
\int_{0}^{\infty} \frac{J(k)(k \cos k y-K \sin k y)}{k^{2}+K^{2}} d k=\frac{\pi}{2} \gamma \exp (-K y)
$$

for $y \leq a$ or $y \geq b$. Thus if $\xi \rightarrow+0, A(+0, \eta)=0$ for $\eta \leq a$ or $\eta \geq b$, and similarly $A(-0, \eta)=0$ for $\eta \leq a$ or $\eta \geq b$. Hence when the source is directly above or beneath the plate, the plate has no effect on the source.

\section{Acknowledgement}

This work is partially supported by a TWAS research scheme (TWAS RG No. 274). The work was done while the author was at the Department of Applied Mathematics, Calcutta University.

\section{References}

[1] U. Basu and B. N. Mandal. "A plane vertical submerged barrier in surface water waves", Internat. J. Math. and Math. Sci. 10 (1987) 815-820.

[2] D. V. Evans, "Diffraction of water waves by a submerged vertical plate", J. Fluid Mech. 40 (1970) 433-451.

[3] D. V. Evans, "A note on the waves produced by the small oscillations of a partially immersed vertical plate", J. Inst. Maths. Applics. 17 (1976) 135-140.

[4] L. S. Gradstein and I. M. Ryzhik, Tables of Integrals, Series and Products (Academic Press, 1980).

[5] M. D. Haskind, "Radiation and diffraction of surface waves by a flat plate floating vertically" (Russ.), Prikl. Mat. Mech. 23 (1959) 546-556.

[6] B. N. Mandal and S. K. Goswami, "The scattering of an obliquely incident surface wave by a vertical plate", J. Math. Phys. 25 (1984) 1780-1783.

[7] F. Ursell, "The effect of a fixed vertical barrier on surface waves in deep water", Proc. Camb. Phil. Soc. 43 (1947) 374-382.

[8] F. Ursell, "On the waves due to rolling of a ship", Q.J. Mech. Appl. Math. 1 (1948) 246-252. 